1. Boyman, O., Conrad, C., Tonel, G., Gilliet, M., and Nestle, F.O. 2007. The pathogenic role of tissueresident immune cells in psoriasis. Trends Immunol. 28:51-57.

2. Clark, R.A., et al. 2006. The vast majority of CLA+ $\mathrm{T}$ cells are resident in normal skin. J. Immunol. 176:4431-4439.

3. Romani, N., et al. 2006. Epidermal Langerhans cells--changing views on their function in vivo. Immunol. Lett. 106:119-125.

4. Nickoloff, B.J. 1993. Dermal immune system. CRC Press. Boca Raton, Florida, USA. 340 pp.

5. Merad, M., et al. 2002. Langerhans cells renew in the skin throughout life under steady-state conditions. Nat. Immunol. 3:1135-1141.

6. Nestle, F.O., Zheng, X.G., Thompson, C.B., Turka, L.A., and Nickoloff, B.J. 1993. Characterization of dermal dendritic cells obtained from normal human skin reveals phenotypic and functionally distinctive subsets. J. Immunol. 151:6535-6545.

7. Lenz, A., Heine, M., Schuler, G., and Romani, N 1993. Human and murine dermis contain dendritic cells. Isolation by means of a novel method and phenotypical and functional characterization. J. Clin. Invest. 92:2587-2596.

8. Nestle, F.O., Filgueira, L., Nickoloff, B.J., and Burg, G. 1998. Human dermal dendritic cells process and present soluble protein antigens. J. Invest. Dermatol. 110:762-766

9. Bogunovic, M., et al. 2006. Identification of a radio-resistant and cycling dermal dendritic cell population in mice and men. J. Exp. Med. 203:2627-2638.

10. Angel, C.E., et al. 2006. Cutting edge: CD1a+ antigen-presenting cells in human dermis respond rap- idly to CCR7 ligands. J. Immunol. 176:5730-5734.

11. Larregina, A.T., et al. 2001. Dermal-resident CD14+ cells differentiate into Langerhans cells. Nat. Immunol. 2:1151-1158.

12. Lowes, M.A., et al. 2005. Increase in TNF-alpha and inducible nitric oxide synthase-expressing dendritic cells in psoriasis and reduction with efalizumab (anti-CD11a). Proc. Natl. Acad. Sci. U. S. A. 102:19057-19062.

13. Nestle, F.O., et al. 2005. Plasmacytoid predendritic cells initiate psoriasis through interferon-alpha production. J. Exp. Med. 202:135-143.

14. Wollenberg, A., et al. 2002. Plasmacytoid dendritic cells: a new cutaneous dendritic cell subset with distinct role in inflammatory skin diseases. J. Invest. Dermatol. 119:1096-1102.

15. Djemadji-Oudjiel, N., Goerdt, S., Kodelja, V., Schmuth, M., and Orfanos, C.E. 1996. Immunohistochemical identification of type II alternatively activated dendritic macrophages (RM 3/1+3, MS 1+/-, 25F9-) in psoriatic dermis. Arch. Dermatol. Res. 288:757-764

16. Weber-Matthiesen, K., and Sterry, W. 1990. Organization of the monocyte/macrophage system of normal human skin. J. Invest. Dermatol. 95:83-89.

17. Zaba, L.C., Fuentes-Duculan, J., Steinman, R.M., Krueger, J.G., and Lowes, M.A. 2007. Normal human dermis contains distinct populations of CD $11 \mathrm{c}^{+} \mathrm{BDCA}^{-1^{+}}$dendritic cells and $\mathrm{CD} 163^{+} \mathrm{FXIIIA}{ }^{+}$ macrophages. J. Clin. Invest. 117:2517-2525. doi:10.1172/JCI32282.

18. Fabriek, B.O., Dijkstra, C.D., and van den Berg, T.K. 2005. The macrophage scavenger receptor CD163. Immunobiology. 210:153-160.

19. Torocsik, D., Bardos, H., Nagy, L., and Adany, R.
2005. Identification of factor XIII-A as a marker of alternative macrophage activation. Cell. Mol. Life Sci. 62:2132-2139.

20. Grassi, F., et al. 1998. Monocyte-derived dendritic cells have a phenotype comparable to that of dermal dendritic cells and display ultrastructural granules distinct from Birbeck granules. J. Lenkoc. Biol. 64:484-493.

21. Caux, C., et al. 1996. CD34+ hematopoietic progenitors from human cord blood differentiate along two independent dendritic cell pathways in response to GM-CSF+TNF alpha. J. Exp. Med. 184:695-706.

22. Cerio, R., Griffiths, C.E., Cooper, K.D., Nickoloff, B.J., and Headington, J.T. 1989. Characterization of factor XIIIa positive dermal dendritic cells in normal and inflamed skin. Br.J. Dermatol. 121:421-431.

23. Gordon, S., and Taylor, P.R. 2005. Monocyte and macrophage heterogeneity. Nat. Rev. Immunol. 5:953-964.

24. Blander, J.M., and Medzhitov, R. 2006. On regulation of phagosome maturation and antigen presentation. Nat. Immunol. 7:1029-1035.

25. Filgueira, L., Nestle, F.O., Rittig, M., Joller, H.I., and Groscurth, P. 1996. Human dendritic cells phagocytose and process Borrelia burgdorferi. J. Immunol. 157:2998-3005.

26. Pozzi, L.A., Maciaszek, J.W., and Rock, K.L. 2005. Both dendritic cells and macrophages can stimulate naive $\mathrm{CD} 8 \mathrm{~T}$ cells in vivo to proliferate, develop effector function, and differentiate into memory cells. J. Immunol. 175:2071-2081.

27. Steinman, R.M., Hawiger, D., and Nussenzweig, M.C. 2003. Tolerogenic dendritic cells. Annu. Rev. Immunol. 21:685-711.

\title{
Taking aim at translation for tumor therapy
}

\author{
Bryan C. Barnhart ${ }^{1}$ and M. Celeste Simon'1,2,3,4
}

${ }^{1}$ Abramson Family Cancer Research Institute, University of Pennsylvania Cancer Center, ${ }^{2}$ Howard Hughes Medical Institute, and ${ }^{3}$ Department of Cancer Biology and ${ }^{4}$ Department of Cell and Developmental Biology, School of Medicine, University of Pennsylvania, Philadelphia, Pennsylvania, USA.

\begin{abstract}
Increased cap-dependent mRNA translation rates are frequently observed in human cancers. Mechanistically, many human tumors often overexpress the cap binding protein eukaryotic translation initiation factor 4E (eIF4E), leading to enhanced translation of numerous tumor-promoting genes. In this issue of the JCI, Graff and colleagues describe potent antitumor effects using second-generation antisense oligonucleotides for $\mathrm{EIF} 4 \mathrm{E}$ (see the related article beginning on page 2638). If their results are recapitulated in a clinical setting, this strategy will provide a promising antitumor therapy with broad-reaching applications.
\end{abstract}

Protein synthesis is required for many critical cellular processes, and cells regulate mRNA translation rates according to their needs. Interestingly, dysregulated translation has now been

Nonstandard abbreviations used: ASO, antisense oligonucleotide; 4E-BP, eIF4E binding protein; eIF4E, eukaryotic translation initiation factor 4E; mTORC1, mammalian target of rapamycin complex 1 .

Conflict of interest: The authors have declared that no conflict of interest exists.

Citation for this article: J. Clin. Invest. 117:2385-2388 (2007). doi:10.1172/JCI33107. linked to multiple human cancers $(1,2)$. Increased translation rates lead to an overproduction of proteins involved in proliferation, survival, metastasis, and other malignant characteristics (3-5). Protein synthesis regulation is complex, and its alteration in tumor cells occurs at numerous points. Many tumor-promoting mechanisms ultimately cause the activation of a critical regulator of capdependent translation, the eukaryotic translation initiation factor 4F (eIF4F) complex. Numerous human tumors exhibit inappropriate eIF4F activation, including lymphomas and breast, prostate, colorectal, head and neck, cervical, bladder, and lung cancers (3). Therefore, therapeutically targeting eIF4F activity is exceedingly attractive, as it would potentially be applicable to a broad range of human cancers. In this issue of the JCI, Graff and colleagues (6) report such a strategy, attacking one of the important components of eIF4F, eIF4E, with striking efficacy in tumor models. If this treatment is successful in the clinic, it holds great promise for use against many human tumors and may be especially effective if used in combination with more traditional chemotherapeutic treatments.

\section{Cap-dependent protein synthesis and its regulation}

To provide better insight into the underlying mechanism for this therapy and why it might ultimately be so effective against 


\section{A Normal tissue}

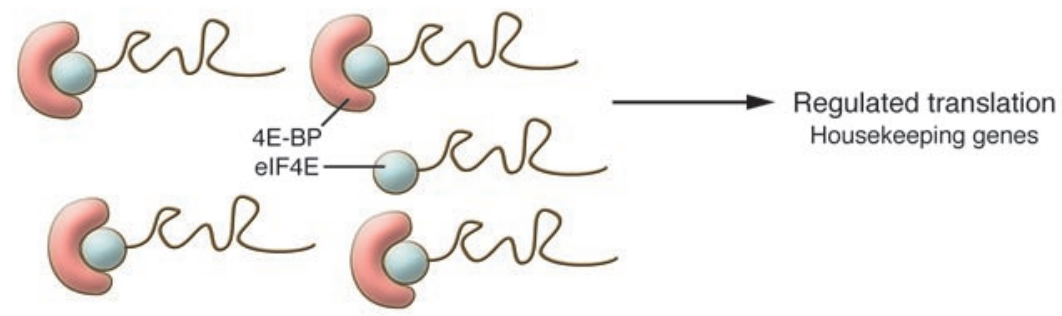

B Tumor

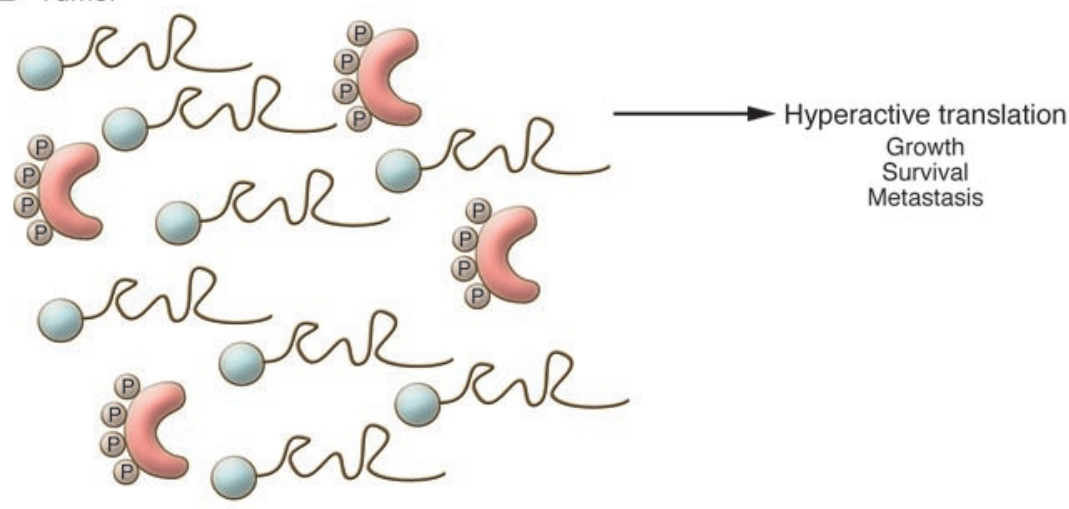

C elF4E ASO-treated tumor
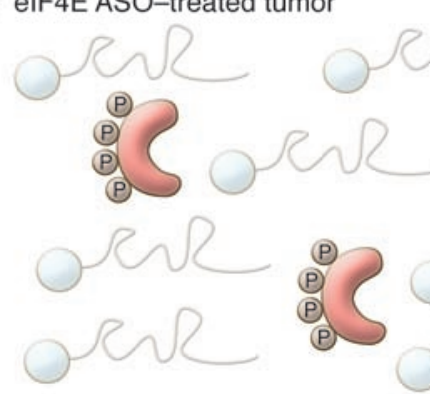

Figure 1

Mechanism of action of eIF4E ASOs. (A) In normal tissues, elF4E is typically sequestered by hypophosphorylated $4 \mathrm{E}-\mathrm{BPs}$, resulting in restricted translation rates. Homeostasis is maintained by limiting translation to essential genes, such as housekeeping genes. (B) In some tumors, oncogenic signaling results in primarily hyperphosphorylated 4E-BPs. Additionally, many tumors express high levels of elF4E. Excess free elF4E leads to increased translation rates, especially of genes involved in proliferation, survival, and metastasis. These increased translation rates help to drive tumor progression. (C) In tumors treated with elF4E ASOs, elF4E levels are significantly reduced. Despite 4E-BP hyperphosphorylation, reduced elF4E levels inhibit translation rates, causing growth arrest or even apoptosis in tumors. It is likely that this strategy would have broad-reaching applications for tumors with elF4E overexpression, oncogenic signaling (leading to 4E-BP hyperphosphorylation), or both. Since tumors frequently rely on increased translation for high proliferation rates and other malignant properties, reducing elF4E levels should have a greater impact on them than it would on normal cells.

cancer, we will briefly describe cap-dependent mRNA translation and its regulation. Translation initiation is primarily controlled by the assembly of two multiprotein complexes and their association with mRNA: the ternary and eIF4F complexes (7). The ternary complex consists of eIF $2 \alpha$, guanosine triphosphate (GTP), and a methionine-charged transfer RNA. It associates with the 40S ribosome and then the 7-methyl-GTP-capped mRNA, which is bound to eIF4F. eIF4F is comprised of the scaffold eIF4G, the ATP-dependent RNA-helicase eIF4A, and the cap binding protein eIF4E. The convergence of these complexes on mRNA allows ribosome scanning and translation initiation. Since eIF4E is the least abundant component, its availability limits translation initiation rates, and therefore translation itself. Free eIF4E levels are determined primarily by their degree of association with a class of regulatory proteins termed eIF4E binding proteins (4E-BPs). 4E-BPs bind to eIF4E at the eIF4G binding site and therefore block eIF4F assembly by sequestering its rate-limiting member. The 4E-BPs (there are three family members) are regulated in turn by phosphorylation at several sites, with increased phosphorylation leading to decreased eIF4E binding capacity. 4E-BP phosphorylation is carried out primarily by the mammalian target of rapamycin complex 1 (mTORC1) (8). mTORC1 gathers information about the cellular environment, including nutrient, growth factor, and oxygen availability and activates downstream effectors accordingly $(2,9) \cdot$ mTORC1 phosphorylates two key substrates, $\mathrm{p} 70^{\mathrm{s} 6 \mathrm{~K}}$, whose activity mediates the binding of several key initiation factors (10), and 4E-BPs. This way, mTORC1 integrates the richness of the environment with biosynthetic pathways downstream, dictating whether cells will grow and proliferate. Importantly, key points in the pathway that activate mTORC1 are frequently mutated in human cancers. For example, PI3K activity is often high in tumors due to the loss of the PTEN tumor suppressor, which allows a constitutive activation of the AKT kinase, inappropriate mTORC1 signaling, and constitutive phosphorylation and inactivation of 4E-BPs (2). Due to the important role of mTORC1 in cell growth and proliferation, multiple mTORC1 inhibitors are progressing through clinical trials.

eIF4E availability dictates not only the rate of protein synthesis but, perhaps more importantly, its quality. It has been proposed that the mRNA 5' untranslated region ( $\left.5^{\prime} \mathrm{UTR}\right)$ structure in part dictates translation efficiency (reviewed in ref. 3). mRNAs with highly complex 5'UTR structures ("weak" mRNAs) are more difficult to translate than those with relatively uncomplicated structures ("strong" mRNAs). This model postulates that strong mRNAs are translated at a relatively constant rate with little impact from active initiation factor availability. Conversely, because weak mRNAs must compete for eIF4E binding and retention, they are translated much more efficiently when eIF4E is present in excess. Importantly, weak mRNAs tend to be those with roles in proliferation (cyclin D1, c-Myc, and ornithine decarboxylase), survival $\left(\mathrm{Bcl}-\mathrm{x}_{\mathrm{L}}, \mathrm{etc}\right.$ ), angiogenesis (VEGF, 
basic FGF, and HIF- $1 \alpha$ ), and other malignancy-promoting functions (MMP9, etc.) (3). Therefore, targeting eIF4F would allow the clinician to hit a variety of gene targets with one therapy, since increased translation rates alter the expression of a variety of genes. In addition, targeting an essential component of translation initiation at a point far downstream should allow little chance of escape via activating upstream components of the pathway. However, as attractive as this strategy is, little success has followed attempts to therapeutically attack the eIF4F complex. First, because eIF4F function is dictated by protein-protein interactions and mRNA binding, complex disruption has proven to be extremely difficult. Additionally, the potential offtarget effects of eliminating an essential component of the translation machinery are troubling. Graff et al. (6) report the use of second-generation antisense oligonucleotides (ASOs) to effectively downregulate eIF4E in tumors, greatly attenuating tumor growth in breast and prostate cancer models. In addition, and perhaps surprisingly, no toxicity was detected using this strategy. These results raise the possibility of effectively attacking protein translation downstream of mTORC1 in the clinic.

\section{Effects of elF4E downregulation in tumors and other tissues}

The use of eIF4E ASOs was previously shown to have the predicted effect of reversing transformation $(11,12)$. However, this strategy was not generally effective due to the difficulties of using ASOs therapeutically. Graff and colleagues (6) utilized second-generation ASOs, which are modified to enhance their tissue half-life. They show that treatment of cells in culture with these oligonucleotides caused a significant decrease in eIF4E expression, leading to decreased expression of cyclin D1 and VEGF (both eIF4E translational targets), without a drop in global protein synthesis. They also demonstrated cytotoxicity in vitro, an important observation in light of the potential antitumor effects of eIF4E ASOs. The most interesting data, however, were generated in vivo: mice with subcutaneous xenograft tumors were treated with eIF4E ASOs, causing a profound reduction in tumor growth. eIF4E ASO administration resulted in an approximately $50 \%$ decrease in eIF4E levels within tumors. This level of downregulation almost completely prevented tumor growth, whereas tumors in control-treated mice continued to grow.
In addition, treatment with eIF4E ASOs downregulated tumor VEGF levels and decreased the overall vessel number within the tumors. The authors then addressed an important question: the potential systemic toxicity that eIF4E downregulation might be predicted to cause. Strikingly, despite a highly significant downregulation (approximately 70\%) of eIF4E in the liver, there was no overall toxicity and no negative effects on liver transaminase levels. It is perhaps somewhat surprising that such a substantial decrease in eIF4E abundance does not negatively impact liver function. Since most housekeeping genes are strong mRNAs, however, low eIF4E levels might be sufficient to translate them (Figure 1). This is a critically important point for the future use and efficacy of eIF4E ASOs, and more testing is certainly in progress to assess its overall effects.

\section{The promises and questions of elF4E therapy in human tumors}

The results presented by Graff et al. (6) are very exciting for human tumor treatment. Some questions remain, however, and must be addressed as this strategy moves forward. It will be necessary to further examine mechanisms of eIF4E ASO function. Clearly, treating cells in vitro with eIF4E ASO induced apoptosis. It is likely that treatment in vivo also causes apoptosis, though this will have to be more carefully examined. Tumors treated with eIF4E ASO did not grow, but neither did they regress, essentially remaining the same size. It will be necessary to determine whether eIF4E ASOs act cytotoxically or cytostatically. It is possible that eIF4E ASOs do not reach a high enough concentration in the xenografts to cause the level of cell death observed in vitro. Alternatively, lowering eIF4E levels in these tumors might simply arrest proliferation. This will be an important point, as the ultimate goal of tumor therapy is the death of tumor cells. If eIF4E ASOs act solely cytostatically, their use as single-agent therapeutics might be limited. However, an approach that could be highly productive might combine eIF4E ASOs with traditional cytotoxic chemotherapeutics. This drug combination would be especially effective if eIF4E ASO treatment lowers the tumor cell apoptotic threshold. In conjunction, these agents together would likely induce massive tumor cell apoptosis.

The ability to treat tumors exhibiting high eIF4E levels is very exciting for several reasons. First, as mentioned above, many tumors have been described in which eIF4E is overexpressed (Figure 1), potentially promising broad application. Furthermore, many tumors, such as PTEN, have mutations in upstream components of the pathway that ultimately regulate eIF4E, and such tumors should be susceptible to therapy aimed downstream of these components. Second, small increases in eIF4E expression have profound effects on tumor promotion (13), increasing the likelihood of eIF4E ASO efficacy. Third, it is known that eIF4E overexpression results in resistance to rapamycin $(14,15)$ and rapamycin/chemotherapy combination (16), so this treatment would offer an alternative for patients with rapamycinresistant tumors. This excitement is not without caveats however. It is also possible that combination therapy (or eIF4E ASO treatment alone) could lower the apoptotic threshold in certain normal tissues. In this scenario, lowering eIF4E levels would have a profoundly deleterious impact on tissues such as the liver, among others, complicating the dosage and benefit. In addition, immune system effects will have to be assessed: multiple immune responses require massive cell proliferation and production of soluble mediators that naturally require the synthesis of new proteins. Thus severely decreasing systemic eIF4E levels might also be detrimental to immune function. Indeed rapamycin, which should inhibit eIF4F activity, was originally used as an immunosuppressive agent, and as such, eIF4E downregulation may have an impact on the efficacy of the immune response. Graff and colleagues assessed splenic mass, which was not changed relative to controls, although these parameters will need to be examined in actively proliferating cells such as those responding to an infection.

\section{Summary and future directions}

Despite these potential hurdles, the data presented by Graff et al. (6) offer exciting and broad-reaching potential for the treatment of many forms of human cancer. Future experiments must address the mechanism(s) by which eIF4E ASOs function. Additionally, potential off-target effects must be assessed, especially in situations in which tissues are stressed or must proliferate. However, this approach holds great promise: attacking the eIF4F complex has been recognized as a potentially excellent therapeutic approach for some time. The difficulty in disrupting this complex therapeutically has made it unproductive in the clinic so far. With the 
development of second-generation ASOs for eIF4E, Graff and colleagues may enable us to finally target this important complex effectively, providing a potentially wide-ranging antitumor modality.

Address correspondence to: M. Celeste Simon, Howard Hughes Medical Institute, Abramson Family Cancer Institute, University of Pennsylvania School of Medicine, BRBII/III, Room 456, 421 Curie Boulevard, Philadelphia, Pennsylvania 19104, USA. Phone: (215) 746-5532; Fax: (215) 746-5511; E-mail: celeste2@mail.med.upenn.edu.

\footnotetext{
1. Pandolfi, P.P. 2004. Aberrant mRNA translation in cancer pathogenesis: an old concept revisited comes finally of age. Oncogene. 23:3134-3137.

2. Bjornsti, M.A., and Houghton, P.J. 2004. The TOR pathway: a target for cancer therapy. Nat. Rev. Cancer. 4:335-348.

3. De Benedetti, A., and Graff, J.R. 2004. eIF-4E expres-
}

sion and its role in malignancies and metastases. Oncogene. 23:3189-3199.

4. Mamane, Y., et al. 2004. eIF4E - from translation to transformation. Oncogene. 23:3172-3179.

5. Bjornsti, M.A., and Houghton, P.J. 2004. Lost in translation: dysregulation of cap-dependent translation and cancer. Cancer Cell. 5:519-523.

6. Graff, J.R., et al. 2007. Therapeutic suppression of translation initiation factor eIF4E expression reduces tumor growth without toxicity. J. Clin. Invest. 117:2638-2648. doi:10.1172/JCI32044.

7. Kapp, L.D., and Lorsch, J.R. 2004. The molecular mechanics of eukaryotic translation. Annu. Rev. Biochem. 73:657-704.

8. Brunn, G.J., et al. 1997. Phosphorylation of the translational repressor PHAS-I by the mammalian target of rapamycin. Science. 277:99-101.

9. Arsham, A.M., Howell, J.J., and Simon, M.C 2003. A novel hypoxia-inducible factor-independent hypoxic response regulating mammalian target of rapamycin and its targets. J. Biol. Chem. 278:29655-29660

10. Holz, M.K., Ballif, B.A., Gygi, S.P., and Blenis, J. 2005. mTOR and S6K1 mediate assembly of the translation preinitiation complex through dynamic protein interchange and ordered phosphorylation events. Cell. 123:569-580

11. Graff, J.R., et al. 1995. Reduction of translation initiation factor 4E decreases the malignancy of rastransformed cloned rat embryo fibroblasts. Int. J. Cancer. 60:255-263.

12. Rinker-Schaeffer, C.W., Graff, J.R., De Benedetti, A., Zimmer, S.G., and Rhoads, R.E. 1993. Decreasing the level of translation initiation factor $4 \mathrm{E}$ with antisense RNA causes reversal of ras-mediated transformation and tumorigenesis of cloned rat embryo fibroblasts. Int. J. Cancer. 55:841-847.

13. Ruggero, D., et al. 2004. The translation factor eIF-4E promotes tumor formation and cooperates with c-Myc in lymphomagenesis. Nat. Med. 10:484-486.

14. Fingar, D.C., et al. 2004. mTOR controls cell cycle progression through its cell growth effectors S6K1 and 4E-BP1/eukaryotic translation initiation factor 4E. Mol. Cell. Biol. 24:200-216.

15. Fingar, D.C., Salama, S., Tsou, C., Harlow, E., and Blenis, J. 2002. Mammalian cell size is controlled by $\mathrm{mTOR}$ and its downstream targets $\mathrm{S} 6 \mathrm{~K} 1$ and 4EBP1/eIF4E. Genes Dev. 16:1472-1487.

16. Wendel, H.G., et al. 2004. Survival signalling by Akt and eIF4E in oncogenesis and cancer therapy. Nature. 428:332-337.

\title{
Two tales concerning skeletal muscle
}

\author{
David J. Glass
}

\author{
Novartis Institutes for BioMedical Research, Cambridge, Massachusetts, USA.
}

\begin{abstract}
It was previously appreciated that the determination of skeletal muscle fiber type (fast or slow) could be regulated by class II histone deacetylases (HDACs), which function by inhibiting the transcription factor myocyte enhancer factor 2 (MEF2). In a report by Potthoff et al. in this issue of the $J C I$, it is further shown that HDACs are degraded via the ubiquitin/proteasome pathway, opening up a search for the putative E3 ligase that mediates the proteolysis of the responsible HDACs (see the related article beginning on page 2459). In a second report, by Suzuki et al., a new convergence between the biology of muscular dystrophy and muscle atrophy is elucidated (see the related study beginning on page 2468). It had previously been known that NO signaling is dysregulated during muscular dystrophy due to the disruption of the dystrophin glycoprotein complex (DGC), which anchors neuronal NOS (nNOS). Here it is shown that $\mathrm{nNOS}$ is similarly perturbed in a setting of skeletal muscle atrophy. Both of these studies suggest new avenues for the treatment of skeletal muscle disease.
\end{abstract}

\section{Ubiquitination of class II HDACs determines slow- versus fast- muscle fiber type}

To the uninitiated, muscle may appear to be homogeneous, but in fact it is com-

Nonstandard abbreviations used: CaMK, calcium/ calmodulin-dependent protein kinase; DGC, dystrophin glycoprotein complex; FoxO, forkhead box O; HDAC, histone deacetylase; MAFbx, muscle atrophy F-box protein; MEF2, myocyte enhancer factor 2; MuRF-1, muscle-specific RING finger protein 1; nNOS, neuronal NOS; SUMO, small ubiquitin-like modifier.

Conflict of interest: D. J. Glass is an employee of Novartis Institutes for BioMedical Research.

Citation for this article: J. Clin. Invest. 117:2388-2391 (2007). doi:10.1172/JCI33379. posed of distinct fiber types, referred to as slow and fast, defined by the myosin isotype expressed in the particular fiber (slow muscle expresses type I myosin; fast fibers can express types IIa, IIb, and IIx) and by the oxidative enzymes that are coexpressed in slow muscle. The variety in fiber type enables the animal to perform different types of work. It had previously been shown that fiber type could be perturbed by class II histone deacetylases (HDACs), which act by repressing the transcription factor myocyte enhancer factor 2 (MEF2), which in turn is required for the transcription of the oxidative genes found in slow fibers (Figure 1) (1).
In a study published in this issue, Potthoff et al. (2) demonstrate that class II HDACs are regulated posttranscriptionally, apparently by the ubiquitin/proteasome pathway; despite being transcribed in soleus muscle, HDAC4 and HDAC5 protein levels were not observed there. However, when mice were treated with an inhibitor of the proteasome - the pharmacologic agent MG132 - an accumulation of HDACs in the soleus muscle was observed. Further, in these experiments on mice, ubiquitinated HDAC species could be isolated from the nuclear fraction of soleus muscle. Thus, in slow but not fast muscle fibers, there is a dearth of HDAC4 and -5 proteins, allowing MEF2 to activate the slow-muscle program (Figure 1).

In the Potthoff et al. study, it was not possible to identify a particular class II HDAC as being necessary and sufficient to regulate MEF2 (2). Studies with knockout animals revealed that HDAC4, -5 , and -9 were able to compensate for each other's absence. It was only when double- and triple-knockout animals were employed that a clear fibertype switch, from fast to slow, was observed (though an HDAC4, -5, -9 triple-knockout animal was not able to improve on the $80 \%$ slow-fiber composition observed in HDAC4, -5 and HDAC5, -9 double knockouts, perhaps indicating that in some fibers there may 